\title{
The mitochondrial genome sequence of the ciliate Paramecium caudatum reveals a shift in nucleotide composition and codon usage within the genus Paramecium
}

\author{
Dana Barth ${ }^{1}$ and Thomas U Berendonk ${ }^{1,2^{*}}$
}

\begin{abstract}
Background: Despite the fact that the organization of the ciliate mitochondrial genome is exceptional, only few ciliate mitochondrial genomes have been sequenced until today. All ciliate mitochondrial genomes are linear. They are $40 \mathrm{~kb}$ to $47 \mathrm{~kb}$ long and contain some 50 tightly packed genes without introns. Earlier studies documented that the mitochondrial guanine + cytosine contents are very different between Paramecium tetraurelia and all studied Tetrahymena species. This raises the question of whether the high mitochondrial $\mathrm{G}+\mathrm{C}$ content observed in $P$. tetraurelia is a characteristic property of Paramecium mtDNA, or whether it is an exception of the ciliate mitochondrial genomes known so far. To test this question, we determined the mitochondrial genome sequence of Paramecium caudatum and compared the gene content and sequence properties to the closely related $P$. tetraurelia.

Results: The guanine + cytosine content of the $P$. caudatum mitochondrial genome was significantly lower than that of $P$. tetraurelia (22.4\% vs. $41.2 \%$ ). This difference in the mitochondrial nucleotide composition was accompanied by significantly different codon usage patterns in both species, i.e. within $P$. caudatum clearly A/T ending codons dominated, whereas for $P$. tetraurelia the synonymous codons were more balanced with a higher number of $\mathrm{G} / \mathrm{C}$ ending codons. Further analyses indicated that the nucleotide composition of most members of the genus Paramecium resembles that of $P$. caudatum and that the shift observed in $P$. tetraurelia is restricted to the P. aurelia species complex.

Conclusions: Surprisingly, the codon usage bias in the P. caudatum mitochondrial genome, exemplified by the effective number of codons, is more similar to the distantly related T. pyriformis and other single-celled eukaryotes such as Chlamydomonas, than to the closely related $P$. tetraurelia. These differences in base composition and codon usage bias were, however, not reflected in the amino acid composition. Most probably, the observed picture is best explained by a hitherto unknown (neutral or adaptive) mechanism that increased the guanine + cytosine content in P. tetraurelia mtDNA on the one hand, and strong purifying selection on the ancestral amino acid composition on the other hand. These contradicting forces are counterbalanced by a considerably altered codon usage pattern.
\end{abstract}

\section{Background}

The genomic information and evolution of the mitochondrial genomes of metazoans is well documented. Knowledge on the evolution of mitochondrial DNA (mtDNA) in single-celled organisms on the other hand is more scarce. In order to promote the understaning of

\footnotetext{
* Correspondence: thomas.berendonk@tu-dresden.de

'University of Leipzig, Chair of Molecular Evolution and Animal Systematics, Talstrasse 33, 04103 Leipzig, Germany

Full list of author information is available at the end of the article
}

the mitochondrial genome evolution of single-celled eukaryotes, it is necessary to fill the large existing gaps of knowledge and data. For example only few ciliate mitochondrial genomes have been sequenced until today, most of them belonging to the genus Tetrahymena: T. pyriformis [1], T. thermophila [2], T. pigmentosa, T. malacensis, T. paravorax [3]. While the newest sequence belongs to Euplotes minuta [4], P. tetraurelia has been among the first unicellular eukaryotes, for which the complete mitochondrial genome sequence

\section{C) Biomed Central}


was determined [5]. Therefore, to fill in one of the above mentioned gaps, we decided to sequence the mitochondrial genome of $P$. caudatum, a close relative of the $P$. aurelia species complex, in order to elucidate the mitochondrial genome evolution within this important ciliate genus.

Two interesting aspects of ciliate mtDNA are predominant and have made the assembly of mitochondrial genomes difficult. First, all hitherto known ciliate mitochondrial genomes are linear, from $40 \mathrm{~kb}$ ( $P$. tetraurelia) to $47 \mathrm{~kb}$ (Tetrahymena) long, and contain some 50 tightly packed genes without introns. Second, for about half of the open reading frames (ORFs) in the ciliate mtDNA, a definite protein function is unknown, because there is not enough similarity to known proteins in other organisms [1,2]. Among these ORFs, ymf77 in the Tetrahymena mtDNA is particularly unusual. This gene is $\sim 1,350$ amino acid residues long, shows extreme sequence divergence among Tetrahymena species and has no homolog in the P. tetraurelia or Euplotes mitochondrial genomes.

Paramecium and Tetrahymena belong to the class Oligohymenophorea, one out of eleven lineages within the phylum Ciliophora. Despite similarities in gene content and genome organization in P. tetraurelia and Tetrahymena mtDNA, there are great differences in the nucleotide composition. All studied Tetrahymena mitochondrial genomes have a guanine and cytosine $(\mathrm{G}+\mathrm{C})$ content of $\sim 20 \%$, whereas in $P$. tetraurelia, the $\mathrm{G}+\mathrm{C}$ content is more than twice as high (c. 41\%, [1]). Paramecium tetraurelia and $T$. thermophila are model organisms, whose macronuclear genome sequences have been recently completed [6,7]. Interestingly, the nuclear $\mathrm{G}+\mathrm{C}$ contents are not significantly different in both species $(69.9 \%$ vs. $72.3 \%$, [8]). This raises the question of whether the high mitochondrial $\mathrm{G}+\mathrm{C}$ content observed in P. tetraurelia is a characteristic property of Paramecium mtDNA, or an exception of the ciliate mitochondrial genomes known so far.

To test this question, we determined the mitochondrial genome sequence of $P$. caudatum and compared its gene content and sequence properties to the closely related $P$. tetraurelia.

\section{Results}

\section{General characterization}

The mitochondrial genome of Paramecium caudatum has been described as a linear molecule of 40-44 kb [9]. We determined 43,660 bp of the genome sequence [GenBank:FN424190], excluding only the terminal repeat regions that usually flank the linear ciliate mtDNA. The overall nucleotide composition was $9.9 \%$ G, $42.7 \% \mathrm{~A}, 34.9 \% \mathrm{~T}$, and $12.5 \% \mathrm{C}$, the $\mathrm{G}+\mathrm{C}$ content thereby being significantly lower than that of $P$. tetraurelia mtDNA (22.4\% vs. $41.2 \%)$. The coding part of the genome was 41,091 bp (94.1\%), the protein-coding part 36,585 bp (83.8\%) in length. We determined 25 protein-coding genes with known function, 17 additional ORFs with unknown function, the small and large subunits of the ribosomal RNA genes and three transfer RNA genes (Figure 1). Genes in the P. caudatum mitochondrial genome were densely packed, with short intergenic spacers (0-85 bp). However, the intergenic regions adjacent to the cox 1 and $c o b$ genes were considerably longer (up to $503 \mathrm{bp}$; Figure 1). An overview of all protein-coding genes and a comparison with the $P$. tetraurelia mtDNA is given in Table 1.

The amino acid sequences of genes with known function were more similar between Paramecium species than sequences of the putative ORFs, with atp 9 and nad10 being the most conserved genes (Table 1). The ratio of nonsynonymous vs. synonymous substitutions $(\mathrm{Ka} / \mathrm{Ks})$ also differed, with $\mathrm{Ka} / \mathrm{Ks}$ values being significantly higher in putative ORFs than in known genes (Mann-Whitney-U-test $\mathrm{z}=-3.30 ; \mathrm{p}<0.001$; mean values of 0.203 and 0.080 , respectively). An exception was the rpl2 gene, which possessed the highest $\mathrm{Ka} / \mathrm{Ks}$ value among all protein-coding genes in Paramecium mtDNA, although it was still well within the range indicative for purifying selection (Table 1).

\section{Nucleotide composition and codon usage}

Nucleotide composition and codon usage of the newly determined $P$. caudatum mitochondrial genome were compared to the previously published mitochondrial genomes of $P$. tetraurelia [GenBank:NC001324] and T. pyriformis [GenBank:NC000862]. In P. caudatum, the G $+\mathrm{C}$ content of the protein-coding genes was similar to the $\mathrm{G}+\mathrm{C}$ content of $T$. pyriformis $(21.7 \%$ and $20.2 \%$, respectively), but much lower than that of $P$. tetraurelia (43.2\%). This difference was even more pronounced at $3^{\text {rd }}$ codon positions, with $56.5 \% \mathrm{G}$ or $\mathrm{C}$ ending codons in P. tetraurelia and only $12.8 \%$ and $11.9 \% \mathrm{G}$ or $\mathrm{C}$ ending codons in $P$. caudatum and $T$. pyriformis, respectively. The $\mathrm{G}+\mathrm{C}$ content of the noncoding regions (intergenic spacers) was slightly lower compared to protein-coding genes in all species, but the relative differences between species were similar (Table 2). In the mitochondrial rRNA genes, on the other hand, the $\mathrm{G}+\mathrm{C}$ content differed only slightly among the ciliates (Table 2).

In order to gain further insight into the nucleotide composition of the Paramecium mtDNA, we sequenced a 776 bp fragment of the Cytochrome oxidase subunit 1 gene (cox1) from 13 more Paramecium species. A phylogenetic tree of Paramecium based on sequences of the cox 1 gene is shown in Figure 2B. The analysis of the nucleotide composition at $3^{\text {rd }}$ codon positions of the 


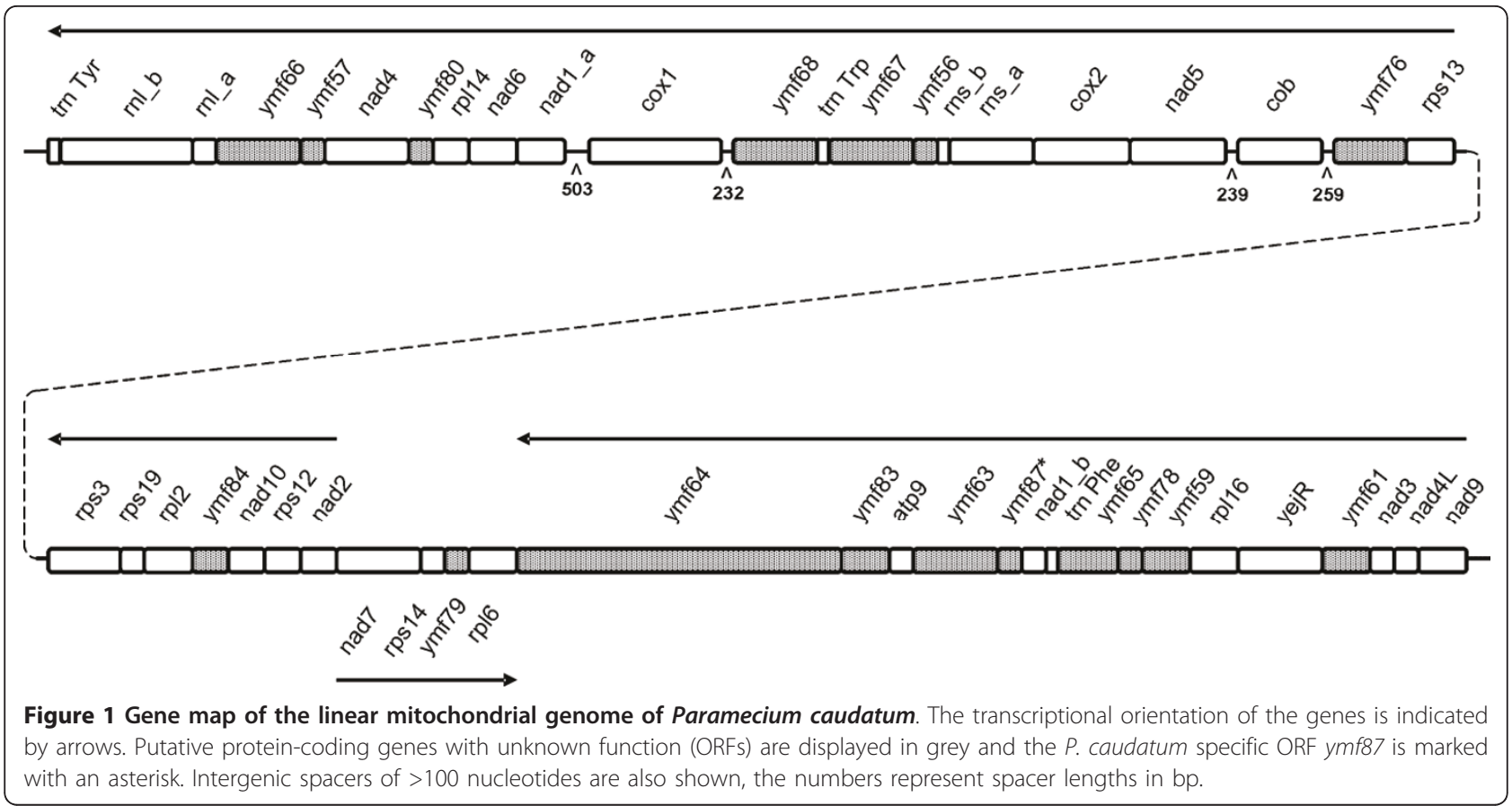

cox1 gene revealed high $\mathrm{G}+\mathrm{C}$ values (45-50\%) in species of the $P$. aurelia complex and a low $\mathrm{G}+\mathrm{C}$ content (3$17 \%$ ) in the remaining paramecia (Figure $2 \mathrm{~A}$ ).

The codon usage bias (CUB) differed significantly between $P$. caudatum and $P$. tetraurelia (Table 3 ). Only in one out of 19 amino acids (Phe) the same codon (TTT) was the most frequently used synonymous codon in $P$. caudatum and $P$. tetraurelia, whereas $P$. caudatum and $T$. pyriformis shared the most frequently used codon in 16 amino acids. The type of preferred codons was related to the nucleotide composition of the respective species (Table 2, 3). Generally, for P. caudatum and T. pyriformis A/T ending synonymous codons were abundant. This was also true for the stop codons, as only TAA was detected to terminate protein-coding genes in $P$. caudatum and Tetrahymena, whereas TAG never occurred (Table 3 ). In P. tetraurelia mtDNA, on the other hand, the codon usage was shifted against G/ $\mathrm{C}$ ending codons. Only in two amino acids (Phe and Trp) the most frequent synonymous codon ended in A or $\mathrm{T}$ (and these are the termination codons). Furthermore, both the number of rare codons and the effective number of codons $(\mathrm{Nc})$ indicate a more balanced usage of synonymous codons in $P$. tetraurelia compared to the other ciliates (Table 3 ). A higher $N c$ in $P$. tetraurelia was found for all protein-coding genes and putative ORFs, except nad9 (additional file 1 Table S1).

\section{Gene content and gene order}

Gene composition, gene order and transcriptional orientation were essentially the same as in P. tetraurelia
$[1,5]$. Most of the few observed differences between $P$. caudatum and $P$. tetraurelia could be attributed to sequencing or annotation errors in the $P$. tetraurelia mtDNA. For example, the small gene rps19 was not described in $P$. tetraurelia neither in the original publication nor in the re-annotation paper $[1,5]$. We determined rps 19 in the newly sequenced $P$. caudatum mitochondrial genome as well as in P. tetraurelia at the same relative position (between rps 3 and $r p l 2$ ) where it is located in all Tetrahymena species $[2,3]$. The mitochondrial genome of $P$. caudatum contained one ORF (ymf87) that was not present in $P$. tetraurelia (Figure 1). Furthermore, we determined five ORFs in P. caudatum, each of which seems to be equivalent to two adjacent ORFs in P. tetraurelia. Four of these ORFs (ymf65, $y m f 66, y m f 67$ and $y m f 76)$ are also present in Tetrahymena mtDNA [1]. The fifth ORF refers to the heme maturase gene (yejR), which was much larger than $P$. tetraurelia yejR (447 vs. 255 aa), but similar to Tetrahymena (512-522 aa) and Euplotes minuta yejR (461 aa) [1-4]. In P. tetraurelia, ymf82 is located directly downstream to the $y e j R$ gene [1]. In $P$. caudatum, however, $y m f 82$ was not present at this position or anywhere else in the mitochondrial genome (Figure 1). These results could indicate sequencing errors in the $P$. tetraurelia mitochondrial genome, which led to several frameshifts and premature stopcodons in the annotated sequence.

The most striking difference compared to $P$. tetraurelia was $y m f 64$, a large open reading frame of $5,136 \mathrm{bp}$ length (Figure 1). The C-terminal part of this ORF showed significant similarity to ymf64 of $P$. tetraurelia 
Table 1 Size of protein-coding genes in $P$. caudatum mtDNA and comparative data calculated for $P$. caudatum and $P$. tetraurelia

\begin{tabular}{|c|c|c|c|c|c|c|c|}
\hline gene & length (in aa) & sequence identity & $\mathrm{Ka} / \mathrm{Ks}$ & ORF & length (in aa) & sequence identity & $\mathrm{Ka} / \mathrm{Ks}$ \\
\hline$c o b$ & 442 & 0.91 & 0.012 & $y m f 56$ & 81 & 0.73 & 0.067 \\
\hline $\operatorname{cox} 1$ & 745 & 0.93 & 0.019 & $y m f 57$ & 105 & 0.77 & 0.069 \\
\hline $\cos 2$ & 577 & 0.84 & 0.022 & ymf59 & 127 & 0.40 & 0.340 \\
\hline $\operatorname{atp9}$ & 75 & 1.00 & 0.001 & ymf61 & 189 & 0.40 & 0.298 \\
\hline yej $R^{a}$ & 447 & 0.47 & 0.242 & $y m f 63^{a}$ & 525 & 0.44 & 0.258 \\
\hline nad1_a & 287 & 0.93 & 0.012 & $y m f 64^{a}$ & 1712 & 0.56 & 0.168 \\
\hline nad1_b & 63 & 0.71 & 0.027 & $y m f 65^{b}$ & 365 & - & - \\
\hline nad2 & 167 & 0.67 & 0.093 & $y m f 66^{b}$ & 412 & - & - \\
\hline nad3 & 120 & 0.83 & 0.033 & $y m f 67^{b}$ & 354 & - & - \\
\hline nad4 & 501 & 0.70 & 0.062 & ymf68 & 395 & 0.76 & 0.044 \\
\hline nad4l & 115 & 0.64 & 0.070 & $y m f 76^{b}$ & 399 & - & - \\
\hline nad5 & 597 & 0.75 & 0.049 & $y m f 78$ & 69 & 0.48 & 0.298 \\
\hline nad6 & 256 & 0.73 & 0.064 & $y m f 79$ & 73 & 0.38 & 0.323 \\
\hline nad7 & 423 & 0.91 & 0.010 & ymf80 & 97 & 0.68 & 0.076 \\
\hline nad9 & 184 & 0.67 & 0.072 & ymf83 & 168 & 0.55 & 0.206 \\
\hline nad10 & 154 & 0.95 & 0.006 & ymf84 & 158 & 0.33 & 0.291 \\
\hline$r p s 3^{a}$ & 363 & 0.57 & 0.195 & $y m f 8 x^{c}$ & 107 & - & - \\
\hline rps 12 & 139 & 0.84 & 0.024 & & & & \\
\hline rps13 & 244 & 0.66 & 0.049 & & & & \\
\hline rps 14 & 102 & 0.59 & 0.104 & & & & \\
\hline $\operatorname{rps} 19^{d}$ & 88 & 0.46 & 0.188 & & & & \\
\hline rpl2 & 265 & 0.68 & 0.523 & & & & \\
\hline rpl6 & 178 & 0.71 & 0.055 & & & & \\
\hline rp/14 & 119 & 0.75 & 0.030 & & & & \\
\hline rp/16 & 166 & 0.74 & 0.047 & & & & \\
\hline
\end{tabular}

The sequence identity values were calculated from amino acid sequences. The Ka/Ks ratio was estimated using the method of Goldman and Yang [47] as implemented in the program Ka/Ks_Calculator [28].

${ }^{a} y e j R, r p s 3, y m f 63$ and $y m f 64$ were considerably longer in the mtDNA of $P$. caudatum. For comparative analyses the sequences were adjusted to the gene length in P. tetraurelia.

b ymf65, ymf66, ymf67 and ymf76 each appear to be equivalent to two adjacent ORFs in P. tetraurelia. Comparative data are omitted.

c ymf87 was only found in $P$. caudatum

d rps19 in P. tetraurelia was annotated during the present work

and Tetrahymena (where this ORF is 234 and 330 amino acids long, respectively) and to the c-terminal part of rps3 in Euplotes. The largest part of the gene, however, consisted of an N-terminal extension. The analysis revealed one transmembrane region from amino acid 1,469 to 1,491 , with the alignable C-terminal part

Table 2 Nucleotide composition in coding and noncoding regions of ciliate mitochondrial genomes

\begin{tabular}{ccccccccccc}
\hline & \multicolumn{3}{c}{ Protein-coding genes } & \multicolumn{3}{c}{ noncoding DNA } & \multicolumn{3}{c}{ rRNA genes } \\
& T. $\boldsymbol{p}$. & P. c. & P. $\boldsymbol{t}$. & T. $\boldsymbol{p}$. & P. c. & P. $\boldsymbol{t}$. & T. $\boldsymbol{p}$. & P. $\boldsymbol{c}$. & P. $\boldsymbol{t}$. \\
\hline $\mathrm{G}$ & 10.3 & 12.1 & 19.5 & 6.2 & 8.0 & 18.7 & 13.8 & 19.0 & 21.2 \\
A & 41.1 & 35.5 & 24.8 & 40.8 & 35.7 & 24.3 & 35.3 & 35.6 & 33.0 \\
T & 38.7 & 42.8 & 32.0 & 46.3 & 49.0 & 36.1 & 35.7 & 32.3 & 30.3 \\
C & 9.9 & 9.6 & 23.7 & 6.7 & 7.3 & 20.9 & 15.2 & 13.1 & 15.5 \\
G+C & $\mathbf{2 0 . 2}$ & $\mathbf{2 1 . 7}$ & $\mathbf{4 3 . 2}$ & $\mathbf{1 2 . 9}$ & $\mathbf{1 5 . 3}$ & $\mathbf{3 9 . 6}$ & $\mathbf{2 9 . 0}$ & $\mathbf{3 2 . 1}$ & $\mathbf{3 6 . 7}$
\end{tabular}

T. p. Tetrahymena pyriformis [GenBank:NC000862], P. c. Paramecium caudatum [GenBank:FN424190], P. t. Paramecium tetraurelia [GenBank:NC001324], values are given in percent. (see above) lying inside and the large extension lying outside of the membrane. BLAST searches of this extension detected no sequence similarity with any known sequence in GenBank.

Besides its size, ymf64 possessed another unusual feature: an intragenic minisatellite, composed of nine repeat units of $18 \mathrm{bp}$ close to the middle of the ORF. We amplified and sequenced this gene region from 20 further $P$. caudatum strains and found the repeat number to be highly variable, even between strains sharing the same $\operatorname{cox} 1$ haplotype (Figure 3). We identified 12 length variants with repeat numbers ranging from six to 23 units. No length polymorphism (heteroplasmy) was detected within individual $P$. caudatum strains. The repeat was imperfect as we found differences (substitutions) between the repeat units within one sequence as well as variations between different strains. However, these sequence variations never led to nonsense mutations. Since the repeat unit size was a multiple of three 


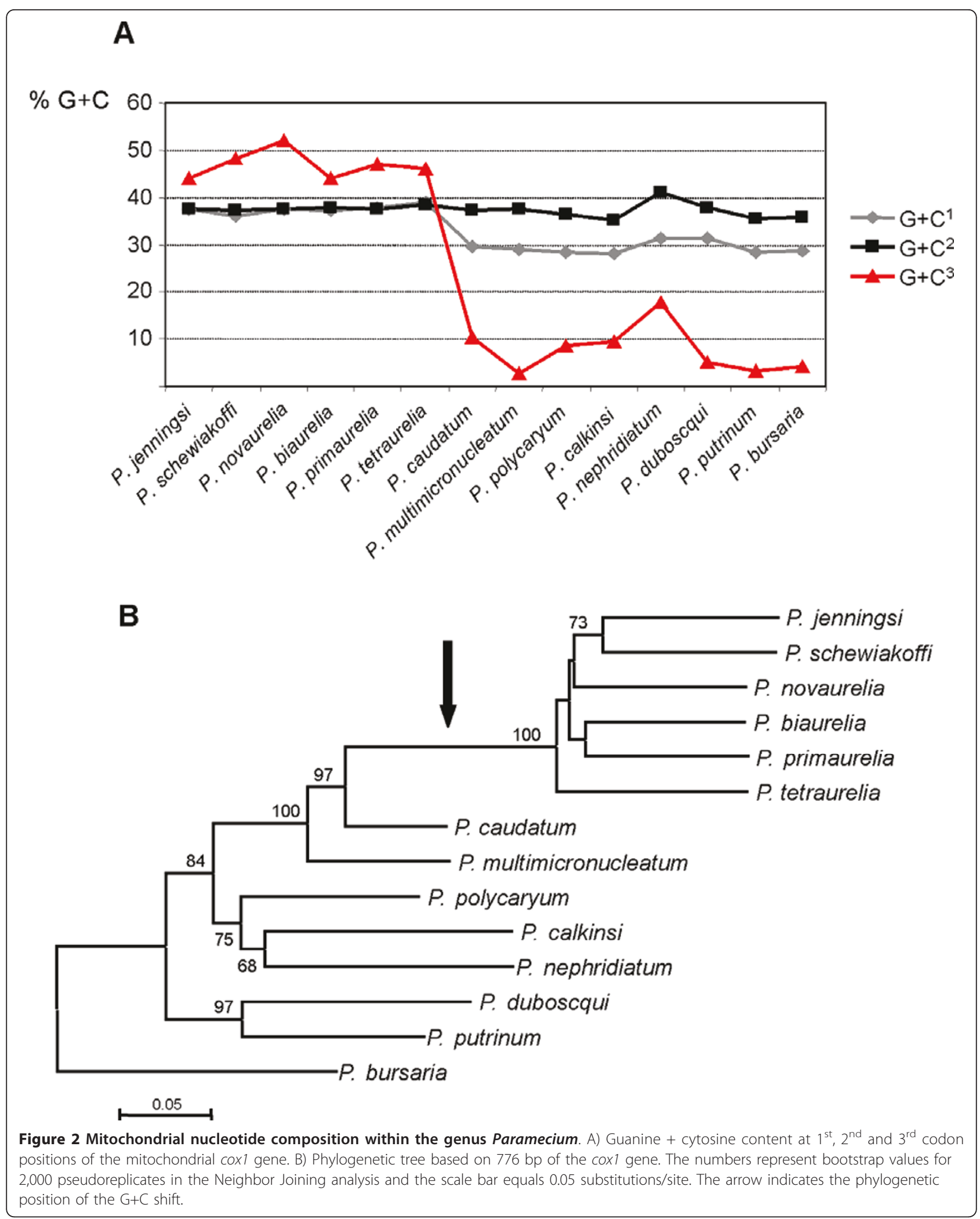


Table 3 Comparative codon usage in the mtDNA of $T$. pyriformis, $P$. caudatum and $P$. tetraurelia

\begin{tabular}{|c|c|c|c|c|c|c|c|c|c|}
\hline & & T.p. & P. c. & P.t. & & & T. p. & P. c. & P.t. \\
\hline \multirow[t]{4}{*}{ Ala } & GCG & 0.03 & 0.03 & 0.08 & Pro & CCG & 0.07 & 0.03 & 0.03 \\
\hline & GCA & 0.32 & 0.41 & 0.20 & & CCA & 0.36 & 0.42 & 0.16 \\
\hline & $\mathrm{GCT}$ & 0.57 & 0.54 & 0.30 & & $\mathrm{CCT}$ & 0.54 & 0.51 & 0.27 \\
\hline & GCC & 0.07 & 0.02 & 0.41 & & CCC & 0.03 & 0.04 & 0.54 \\
\hline \multirow[t]{2}{*}{ Cys } & TGT & 0.86 & 0.85 & 0.24 & Gln & CAG & 0.06 & 0.14 & 0.58 \\
\hline & TGC & 0.14 & 0.15 & 0.76 & & CAA & 0.94 & 0.86 & 0.42 \\
\hline \multirow[t]{3}{*}{ Asp } & GAT & 0.81 & 0.81 & 0.36 & Arg & AGG & 0.02 & 0.17 & 0.45 \\
\hline & GAC & 0.19 & 0.19 & 0.64 & & AGA & 0.95 & 0.74 & 0.16 \\
\hline & & & & & & CGG & 0.00 & 0.00 & 0.03 \\
\hline \multirow[t]{3}{*}{ Glu } & GAG & 0.08 & 0.17 & 0.70 & & CGA & 0.00 & 0.02 & 0.11 \\
\hline & GAA & 0.92 & 0.83 & 0.30 & & CGT & 0.02 & 0.06 & 0.06 \\
\hline & & & & & & CGC & 0.00 & 0.01 & 0.18 \\
\hline \multirow[t]{3}{*}{ Phe } & $\pi T$ & 0.89 & 0.95 & 0.62 & & & & & \\
\hline & TTC & 0.11 & 0.05 & 0.38 & Ser & AGT & 0.21 & 0.24 & 0.07 \\
\hline & & & & & & AGC & 0.06 & 0.09 & 0.27 \\
\hline \multirow[t]{4}{*}{ Gly } & GGG & 0.03 & 0.05 & 0.27 & & TCG & 0.03 & 0.03 & 0.12 \\
\hline & GGA & 0.16 & 0.19 & 0.20 & & TCA & 0.32 & 0.20 & 0.10 \\
\hline & GGT & 0.78 & 0.73 & 0.16 & & $\mathrm{TCT}$ & 0.34 & 0.42 & 0.24 \\
\hline & GGC & 0.03 & 0.04 & 0.37 & & TCC & 0.04 & 0.03 & 0.20 \\
\hline \multirow[t]{3}{*}{ His } & CAT & 0.69 & 0.69 & 0.31 & Thr & ACG & 0.01 & 0.03 & 0.22 \\
\hline & CAC & 0.31 & 0.31 & 0.69 & & $\mathrm{ACA}$ & 0.53 & 0.45 & 0.18 \\
\hline & & & & & & $\mathrm{ACT}$ & 0.41 & 0.49 & 0.23 \\
\hline \multirow[t]{4}{*}{ Ile } & ATA & 0.62 & 0.42 & 0.21 & & ACC & 0.06 & 0.03 & 0.37 \\
\hline & ATT & 0.33 & 0.53 & 0.33 & & & & & \\
\hline & ATC & 0.06 & 0.05 & 0.46 & Val & GTG & 0.04 & 0.06 & 0.19 \\
\hline & & & & & & GTA & 0.49 & 0.35 & 0.20 \\
\hline \multirow[t]{2}{*}{ Lys } & AAG & 0.04 & 0.11 & 0.64 & & GTT & 0.42 & 0.55 & 0.30 \\
\hline & AAA & 0.96 & 0.89 & 0.36 & & GTC & 0.06 & 0.04 & 0.31 \\
\hline \multirow[t]{7}{*}{ Leu } & TTG & 0.03 & 0.09 & 0.13 & Trp & TGG & 0.03 & 0.01 & 0.48 \\
\hline & TTA & 0.75 & 0.74 & 0.14 & & TGA & 0.97 & 0.99 & 0.52 \\
\hline & $\mathrm{CTG}$ & 0.01 & 0.01 & 0.09 & & & & & \\
\hline & CTA & 0.13 & 0.05 & 0.15 & Tyr & TAT & 0.82 & 0.79 & 0.38 \\
\hline & $\mathrm{CTT}$ & 0.07 & 0.11 & 0.19 & & TAC & 0.18 & 0.21 & 0.62 \\
\hline & $\mathrm{CTC}$ & 0.01 & 0.01 & 0.31 & & & & & \\
\hline & & & & & Stop & TAG & 0.00 & 0.00 & 0.39 \\
\hline Met & ATG & 1.00 & 1.00 & 1.00 & & TAA & 1.00 & 1.00 & 0.61 \\
\hline \multirow[t]{2}{*}{ Asn } & AAT & 0.79 & 0.77 & 0.34 & & rare & 28 & 23 & 5 \\
\hline & AAC & 0.21 & 0.23 & 0.66 & & Nc & 31.5 & 33.5 & 52.1 \\
\hline
\end{tabular}

Bold values indicate the most frequent synonymous codons for each amino acid and species, italic values indicate rare codons. The overall number of rare codons (rare) and the effective number of codons $(\mathrm{Nc}$ ) are also given for each species.

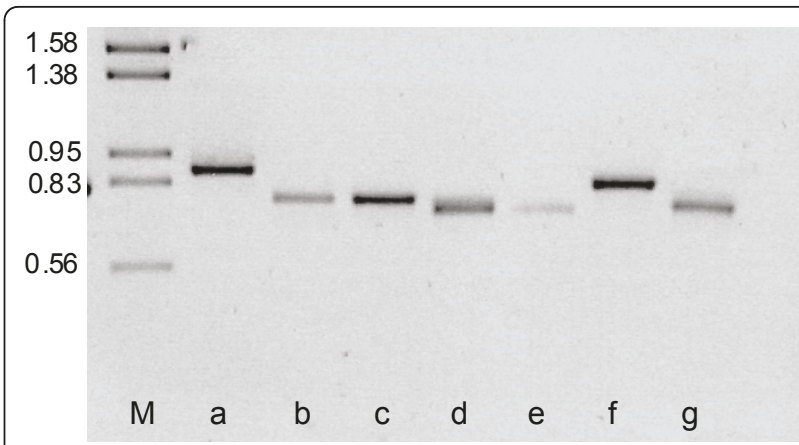

Figure 3 Tandem repeat region in the open reading frame ymf64 amplified from different Paramecium caudatum strains. DNA fragments from strains belonging to the same cox 1 haplotype PcCOI_a03 [35] were separated on a 2.0\% agarose gel. M, DNA size ladder in kb; lanes $a-g$, PCR amplified fragments from the different strains. Number of repeat units: (a) 18; (b) 11 ; (c) 11; (d) 9; (e) 9; (f) $14 ;(g) 10$

nucleotides, length variation in the repeat region did not cause a frameshift in the gene and left the reading frame intact.

\section{Discussion}

\section{Codon usage}

Closely related species usually show similar nucleotide composition and codon usage patterns. For example, five Tetrahymena species possess very similar mitochondrial G+C contents $(18.5 \%-21.3 \%$; [3]), whereas T. pyriformis, $P$. tetraurelia and $E$. minuta differ significantly in this respect $(21.7 \%, 41.9 \%$ and $36.0 \%$, respectively; $[1,4])$. Therefore, it was unexpected that nucleotide composition and codon usage of $P$. caudatum mtDNA were much more similar to Tetrahymena than to the congeneric $P$. tetraurelia.

Phylogenetic relationships within the genus Paramecium are well studied $[10,11]$. In a species phylogeny based on $18 \mathrm{~S}$ rRNA data, $P$. caudatum and the $P$. aurelia species complex are closely related and belong to the "P. aurelia subgroup" [11]. This was also supported by the tree based on cox 1 sequences (Figure 2B). Taken into consideration the phylogenetic position of $P$. tetraurelia, it seems reasonable to hypothesize that a low $\mathrm{G}$ $+\mathrm{C}$ content and the preference for $\mathrm{A} / \mathrm{T}$ ending codons represent the ancestral state in the genus Paramecium. An increased $\mathrm{G}+\mathrm{C}$ content in combination with the preference for $\mathrm{G} / \mathrm{C}$ ending codons would be a derived condition in P. aurelia mtDNA. No further complete Paramecium mitochondrial genomes were available to test this hypothesis. We analyzed the nucleotide 
composition in a fragment of the $\operatorname{cox} 1$ gene, which can be used as an indicator for the overall mitochondrial $G$ $+C$ content $[12,13]$. These sequences supported the above hypothesis, since the shift in the nucleotide composition obviously took place in the lineage leading to the $P$. aurelia species complex (Figure 2A, B). Of course, this $\operatorname{cox} 1$ data set contained only few of the 15 known $P$. aurelia species, and for a conclusive statement all relevant species should be included. But in this context, please note that [14] and [15] obtained DNA sequences from different mitochondrial genes of all $P$. aurelia species and these data suggest that the increased mitochondrial $\mathrm{G}+\mathrm{C}$ content is a consistent pattern throughout the species complex. The observed shift in the nucleotide composition of $P$. tetraurelia mtDNA was associated with a significantly reduced CUB. The CUB in $P$. caudatum mtDNA, exemplified by the effective number of codons, was similar to T. pyriformis and other singlecelled eukaryotes such as Chlamydomonas [16]. P. tetraurelia, on the other hand, had a much lower CUB (i.e. higher $N c$ ) in nearly all genes (Table 3 , additional file 1 , Table S1). These differences in base composition and CUB were not reflected in the derived amino acid composition, which was similar for all the compared species (additional file 2, Figure S1). This suggests strong purifying selection on the mitochondrial amino acid composition in $P$. tetraurelia.

The causes for this large shift in $\mathrm{G}+\mathrm{C}$ content and codon usage in $P$. tetraurelia mtDNA are unknown. In a recent publication, the complete macronuclear genome sequences of $P$. tetraurelia and $T$. thermophila were compared [8]. The authors observed very similar nucleotide composition and codon usage patterns in both species. Thus, whatever caused the shift in the $P$. tetraurelia mitochondrial genome seems to have only affected mtDNA and not nuclear DNA. Furthermore, the altered nucleotide composition was observed for both protein-coding and noncoding regions (Table 2), indicating that the increased $\mathrm{G}+\mathrm{C}$ content is not the consequence of selection for either a higher expression level of $P$. tetraurelia mitochondrial genes, or towards certain codons. Possible explanations for the increased $\mathrm{G}+\mathrm{C}$ content in the $P$. tetraurelia mitochondrial genome are discussed below.

A high $\mathrm{G}+\mathrm{C}$ content could be an adaptation to temperature, UV exposure or other environmental conditions that require a high DNA stability [17] (but see [18]). Although the presently available data suggest that some $P$. aurelia species may be restricted to certain biogeographic regions $[19,20]$, there is no evidence for $P$. tetraurelia generally preferring higher temperatures than $P$. caudatum. In fact, both species often co-occur and have to cope with similar environmental conditions [21].
The observed pattern in P. tetraurelia mtDNA could as well be the consequence of neutral processes like biased gene conversion (BGC) or a general change in mutation pressure from AT towards GC. A neutral process being the underlying mechanism is in concordance with the observation that the $\mathrm{G}+\mathrm{C}$ content in $P$. tetraurelia mtDNA was highest at most neutrally evolving sites ( $3^{\text {rd }}$ codon positions and noncoding regions). In more constrained $m t D N A$ regions $\left(2^{\text {nd }}\right.$ codon positions and ribosomal RNA genes), the base composition of $P$. tetraurelia was not significantly different from $P$. caudatum (Table 2, Figure 2). However, this observation is not per se evidence for neutrality of the underlying mechanism. It is in principle also consistent with an adaptive process in combination with strong selective pressure on the amino acid composition.

$\mathrm{BGC}$ is a $\mathrm{GC}$ biased repair process in genome regions that undergo recombination and has been identified as a major drive in genome evolution [22]. Gene conversion has been suggested as recombinational mechanism in the mitochondrial genome of the killifish Kryptolebias marmoratus [23], and as an explanation for the exceptionally high mitochondrial $\mathrm{G}+\mathrm{C}$ content in the green alga Polytomella capuana [24]. Previous studies using mtDNA found no evidence for recombination events among $P$. aurelia species $[14,15]$. It is, nevertheless, possible that such a mechanism has played a role in the evolutionary history of the $P$. aurelia complex.

In this context it is interesting to note, that a whole genome duplication (WGD) has occurred in the common ancestor of the $P$. aurelia complex [6]. A significantly reduced CUB was also found in nuclear encoded mitochondrial genes of Saccharomyces species, which experienced a WGD [25]. To our knowledge, the influence of a WGD on the mitochondrial genome has not been investigated. It is tempting to speculate that the WGD influenced the crosstalk between nuclear and mitochondrial genomes (e.g. through nuclear encoded genes for the mitochondrial replication and repair machinery).

In conclusion, the observed picture is best explained by a hitherto unknown (neutral or adaptive) mechanism that increased the $\mathrm{G}+\mathrm{C}$ content in $P$. aurelia mtDNA on the one hand, and strong purifying selection on the ancestral amino acid composition on the other hand. These contradicting forces are counterbalanced by a considerably altered codon usage pattern.

\section{Gene content and sequence evolution compared to $P$. tetraurelia}

The size of the P. caudatum mitochondrial genome was within the range predicted from earlier RFLP analyses [9] and $\sim 10 \%$ longer than the mitochondrial genome of $P$. tetraurelia [5]. This difference was primarily due to 
the presence of the large open reading frame $y m f 64$ in $P$. caudatum (discussed in more detail below).

We found one unique ORF (ymf87) in $P$. caudatum, which was not present in P. tetraurelia. This is in contrast to Tetrahymena, where complete synteny among five phylogenetically diverse Tetrahymena species was observed, except for some rare gene duplications [3].

The existence of many ORFs of unknown function is a general problem in protist mitochondrial genomes [26]. Likewise, for about half of the protein-coding mitochondrial genes in ciliates no definite function can be assigned [1-3]. One main objective of studies obtaining new ciliate mitochondrial genomes is therefore the annotation of these unknown $y m f$ genes. Unfortunately we failed to assign any function to hitherto unknown genes, which is most likely due to the high evolutionary rate of ciliate mtDNA. This problem even challenged the alignment of some ORFs from the closely related $P$. caudatum and $P$. tetraurelia. For example, in ymf 84 only $33 \%$ of the amino acids were identical among both species. Therefore, BLAST searches as well as manual alignments could not determine a possible equivalent of this gene in Tetrahymena. The authors of the T. pyriformis mtDNA study suggested a possible homology of Paramecium ymf84 and T. pyriformis ymf 74 based on gene size and position (between nad10 and rpl2) [1]. The recently published mitochondrial genome sequence of the rather distantly related Euplotes minuta confirmed this suggestion, as this conserved gene order was also found in Euplotes [4].

In general, evolutionary rates are governed by negative (purifying) selection, neutrality or positive (diversifying) selection. The ratio of nonsynonymous and synonymous substitution rates $(\mathrm{Ka} / \mathrm{Ks})$ allows an estimation of the selective constraint on a given gene. Values $<1$ are indicative for purifying selection, whereas values $>1$ indicate diversifying selection $[27,28]$. $\mathrm{Ka} / \mathrm{Ks}$ values of the protein-coding genes in the Paramecium mtDNA were generally $<1$, indicating that purifying selection is acting on all genes.

The genes with high sequence similarities between $P$. caudatum and $P$. tetraurelia also had low $\mathrm{Ka} / \mathrm{Ks}$ values. This may indicate that the selective optimum for those genes is similar in the compared species. An exception to this trend was the ribosomal protein gene $r p l 2$, whose $\mathrm{Ka} / \mathrm{Ks}$ ratio indicates a relaxed selective constraint in combination with moderate sequence divergence. In Tetrahymena, no elevated $\mathrm{Ka} / \mathrm{Ks}$ ratios were observed for this gene $[2,3]$. In the present study, putative ORFs had significantly (2.5fold) higher $\mathrm{Ka} / \mathrm{Ks}$ values compared to known protein-coding genes, indicating a lower selective pressure on those non-annotated genes. Similar results were obtained in a study comparing $T$. pyriformis and T. thermophila [2]. Furthermore, these authors supposed that the high divergence of putative ORFs causes difficulties to detect homologous genes through similarity searches. Some of the putative ORFs in Paramecium, however, had low $\mathrm{Ka} / \mathrm{Ks}$ values and a relatively high sequence similarity in $P$. caudatum and $P$. tetraurelia (Table 1). It is surprising that for these genes, which are conserved within ciliates and seem to be under notable selective pressure, no homologs could be detected in other organisms.

\section{Ymf64}

The most conspicuous feature in the P. caudatum mtDNA was a large N-terminal extension of ymf64. compared to the previously published ciliate mitochondrial genomes. This was also the main reason for the length difference between the mitochondrial genomes of P. tetraurelia and P. caudatum (40,469 vs. 43,660 bp). An initial PCR survey revealed that the large gene extension was not present in the mitochondrial genomes of three further members of the $P$. aurelia complex $(P$. primaurelia, $P$. pentaurelia and $P$. jenningsi), $P$. multimicronucleatum, $P$. nephridiatum and $P$. putrinum (data not shown). These results together with the phylogenetic analysis (Figure 2B) suggest that this gene extension is a unique feature of $P$. caudatum rather than an ancestral feature that was lost in P. tetraurelia. The Cterminal part of $y m f 64$ also showed similarity to the Cterminal part of a gene that was annotated as rps3 in Euplotes (but is not homologous to rps3 in Paramecium and Tetrahymena mtDNA). Interestingly, also in Euplotes this gene possesses a large $\mathrm{N}$-terminal extension, although it is smaller than in $P$. caudatum (gene length of 758 aa in E. minuta) [4]. The size of $y m f 64$ resembles $y m f 77$ in the Tetrahymena mtDNA, which is approximately 1,300 amino acids long [2]. However, besides the gene length no other similarities could be detected. The existence of two remarkably large but unrelated mitochondrial genes in two relatively closely related ciliate genera is surprising and needs further study. We are currently investigating the sequence and length variation of ymf64 in $P$. caudatum and will furthermore apply RT-PCR analyses to test whether the gene is expressed in full length.

The length variable repeat in the middle of $y m f 64$ is the first report of an intragenic mitochondrial minisatellite in ciliates. Variable Number Tandem Repeats (VNTRs) or minisatellites are common in the mtDNA of many different organisms, especially metazoans [29]. The high variability (i.e. frequent changes in the number of repeat units) is supposed to occur through slipped strand mispairing during mtDNA replication. In most cases these VNTRs are located in or adjacent to the 
noncoding mitochondrial control region. To our knowledge, an intragenic mitochondrial minisatellite has been reported only from a gene of unknown function in the oomycete Phytophthora sojae [30]. On the other hand, minisatellites encoding nuclear sequences are not unusual. Genes containing polymorphic repeat elements are capable to rapidly adapt to changing environmental conditions or to generate new functions. This is of special importance in developmental genes of multicellular organisms [31] and in cell wall/surface proteins of unicellular prokaryotes [32] and eukaryotes [33] Based on the present data, however, it is not possible to suggest a potential function for the repeat region within $y m f 64$.

Minisatellites have been useful for the investigation of population structure and phylogeographic patterns and also for the identification of individual organisms or clones [34]. Only recently, mtDNA sequences have been successfully applied as genetic markers for the investigation of intraspecific variation in ciliates $[35,36]$. In one of these studies several polymorphic cox 1 haplotypes were observed among natural isolates of $P$. caudatum and $P$. multimicronucleatum, which reflected no clear geographic structure [35]. The ymf64 repeat region proved to be polymorphic in different $P$. caudatum isolates that share the same $\operatorname{cox} 1$ haplotype (Figure 3 ). This indicates a higher evolutionary rate of this sequence region. Further analyses including more isolates will show whether the $y m f 64$ minisatellite has potential as a genetic marker for Paramecium.

\section{Conclusions}

The aim of this study was to compare the mitochondrial genome sequence of Paramecium caudatum to the previously published mtDNA of P. tetraurelia. While gene order and gene content were very similar in both species, the analysis revealed that nucleotide composition and codon usage bias of the $P$. caudatum mtDNA differed greatly from $P$. tetraurelia. The $\mathrm{G}+\mathrm{C}$ content of the $P$. tetraurelia mtDNA was nearly twice as high as in $P$. caudatum (41.2\% vs. $22.4 \%$ ) and the codon usage bias was much lower ( $\mathrm{Nc}=: 52.1$ vs. 33.5). Unexpectedly, the effective number of codons in the P. caudatum mitochondrial genome, is more similar to the distantly related $T$. pyriformis and other single-celled eukaryotes such as Chlamydomonas, than to the closely related $P$. tetraurelia. The analysis of cox 1 data from additional Paramecium species showed that the shift towards a higher mitochondrial $\mathrm{G}+\mathrm{C}$ content took place in the lineage leading to the $P$. aurelia species complex. The reasons for this shift are presently unknown and future studies will investigate whether this is linked to the whole genome duplication that occurred in the common ancestor of the $P$. aurelia complex.

\section{Methods}

\section{Paramecium strains}

The $P$. caudatum strain GB-E used for mitochondrial genome sequencing was originally isolated from a small pond in the vicinity of the University of Edinburgh and kept as clonal culture in the collection of the Molecular Evolution laboratory at Leipzig University. The following Paramecium species were used for the nucleotide composition analysis and phylogenetic reconstruction (Accession numbers for the cox 1 sequences are given in parentheses): P. primaurelia [GenBank:FN421324], $P$. biaurelia [GenBank:FN421325], P. tetraurelia [GenBank: FN421326], P. novaurelia [GenBank:FN421327], P. jenningsi [GenBank:FN421328], P. schewiakoffi [GenBank: AM072773]), P. multimicronucleatum [GenBank: AM072766], P. calkinsi [GenBank:FN421329], P. nephridiatum [GenBank:FN421331], P. duboscqui [GenBank: FN421332], $P$. polycaryum [GenBank:FN421330], $P$. putrinum [GenBank:FN421333], P. bursaria [GenBank: FN421334].

\section{Molecular methods}

DNA extraction followed a Chelex ${ }^{\circledR}$ protocol as described in [35]. The nearly complete mitochondrial genome of $P$. caudatum was amplified in overlapping fragments of 3-15 kb length using Phusion ${ }^{\mathrm{TM}}$ high fidelity DNA polymerase (Finnzymes OY). PCR primers were designed from alignments of conserved gene regions from $P$. tetraurelia [GenBank:NC001324] and $T$. pyriformis [GenBank:NC000862].

PCR products $<5 \mathrm{~kb}$ were directly sequenced employing a primer walking method. Longer PCR products were gel-purified (GFX ${ }^{\mathrm{TM}}$ PCR DNA and Gel Band Purification Kit, Amersham Biosciences) and then digested with the restriction endonuclease $\mathrm{XbaI}$ (Fermentas) to obtain smaller fragments. Restriction fragments up to 3 kb were A-tailed using a Taq DNA polymerase (Fermentas), cloned into pGEM-T Vector (Promega), and sequenced with universal vector primers. Longer fragments were completed by primer walking. Peripheral regions of the linear mtDNA were amplified via step-out PCR [37], single primer PCR [38], and uneven PCR [39]. Cox 1 fragments were amplified with primers and PCR conditions described in [35].

\section{Genome annotation and sequence analysis}

Sequences were manually checked and assembled using BioEdit v. 7.0.5.3 [40] and then annotated with ARTEMIS v. 9 [41]. Open reading frames of $>60$ aa length were compared to GenBank entries using the BlastP program [42]. Additionally, the corresponding nucleotide sequences and intergenic spacers supposed to contain the RNA genes were manually aligned to the 
mitochondrial genome of $P$. tetraurelia [GenBank: NC001324]. The tRNAscan-SE server [43] was employed to assign the tRNA genes. The $\mathrm{Ka} / \mathrm{Ks}$ _Calculator [28] was used to estimate the selective pressure on proteincoding genes. Phylogenetic analyses were carried out with MEGA4 [44]. Nucleotide composition, codon usage, and sequence statistics were analyzed with MEGA4 and different programs implemented in the Sequence Manipulation Suite [45]. The effective number of codons $(\mathrm{Nc})$ was estimated with the program CodonW v. $1.3 \mathrm{http}: / /$ codonw.sourceforge.net// and the number of rare codons was calculated according to [8]. $N c$ describes to what degree the codon usage in a genome is biased. It is a number between 20 and 61 where 20 means only one codon is used for each amino acid and values approaching 61 suggest equal usage of synonymous codons [46].

\section{Additional material}

Additional file 1: Codon usage and open reading frames in Paramecium. Effective number of codons Nc in protein-coding genes and putative ORFs of Paramecium tetraurelia and $P$. caudatum mtDNA.

Additional file 2: Amino acid composition of the mitochondrial genome. Amino acid composition in the mitochondrial genomes of Paramecium caudatum, P. aurelia and Tetrahymena pyriformis (values are given in percent).

\section{Acknowledgements}

This study was supported by a grant from Deutsche

Forschungsgemeinschaft (DFG), priority programme "AQUASHIFT" (BE 2299/ $3-1,2,3)$ and the priority programme "Host-Parasite Coevolution - Rapid Reciprocal Adaptation and its Genetic Basis" (BE 2299/5-1). We thank Tom Little for collecting the P. caudatum strain in Edinburgh, S. I. Fokin for providing the strains of $P$. calkinsi, $P$. jenningsi and $P$. duboscqui, and $C$. Burkhart for her help in sequencing the repeat region of some $P$. caudatum strains. We thank A. Dorothea Sommerfeldt for her very helpful corrections of our manuscript. Finally we thank two anonymous reviewers for careful suggestions, which improved the manuscript considerably.

\section{Author details}

${ }^{1}$ University of Leipzig, Chair of Molecular Evolution and Animal Systematics, Talstrasse 33, 04103 Leipzig, Germany. ㄹesden University of Technology, Institute for Hydrobiology, Zellescher Weg 40, 01062 Dresden, Germany

\section{Authors' contributions}

DB conducted the experiments and performed all necessary analyses, TUB wrote the grant proposal and assisted in the design of the experiments. Both authors wrote the manuscript and approved the final version.

Received: 21 December 2010 Accepted: 31 May 2011 Published: 31 May 2011

\section{References}

1. Burger G, Zhu Y, Littlejohn TG, Greenwood SJ, Schnare MN, Lang BF, Gray MW: Complete sequence of the mitochondrial genome of Tetrahymena pyriformis and comparison with Paramecium aurelia mitochondrial DNA. J Mol Biol 2000, 297:365-380.

2. Brunk $C F$, Lee $L C$, Tran $A B$, Li JL: Complete sequence of the mitochondrial genome of Tetrahymena thermophila and comparative methods for identifying highly divergent genes. Nucleic Acids Res 2003, 31:1673-1682.

3. Moradian MM, Beglaryan D, Skozylas JM, Kerikorian V: Complete mitochondrial genome sequence of three Tetrahymena species reveals mutation hot spots and accelerated nonsynonymous substitutions in ymf genes. PLOS ONE 2007, 2:e650.

4. De Graaf RM, van Alen TA, Dutilh BE, Kuiper JWP, van Zoggel HJAA, Huynh MB, Görtz H-D, Huynen MA, Hackstein JHP: The mitochondrial genomes of the ciliates Euplotes minuta and Euplotes crassus. BMC Genomics 2009, 10:514

5. Pritchard AE, Seilhamer JJ, Mahalingam R, Sable CL, Venuti $\mathrm{SE}$ Cummings DJ: Nucleotide sequence of the mitochondrial genome of Paramecium. Nucleic Acids Res 1990, 18:173-180.

6. Aury JM, Jaillon O, Duret L, Noel B, Jubin C, Porcel BM, Ségurens B, Daubin V, Anthouard V, Aiach N, Arnaiz O, Billaut A, Beisson J, Blanc I, Bouhouche K, Câmara F, Duharcourt S, Guigo R, Gogendeau D, Katinka M, Keller AM, Kissmehl R, Klotz C, Koll F, Le Mouël A, Lepère G, Malinsky S, Nowacki M, Nowak JK, Plattner H, Poulain J, Ruiz F, Serrano V, Zagulski M, Dessen P, Bétermier M, Weissenbach J, Scarpelli C, Schächter V, Sperling L, Meyer E, Cohen J, Wincker P: Global trends of whole-genome duplications revealed by the ciliate Paramecium tetraurelia. Nature 2006 444:171-178.

7. Eisen JA, Coyne RS, Wu M, Wu D, Thiagarajan M, Wortman JR, Badger JH, Ren Q, Amedeo P, Jones KM, Tallon LJ, Delcher AL, Salzberg SL, Silva JC, Haas BJ, Majoros WH, Farzad M, Carlton JM, Smith RK Jr, Garg J, Pearlman RE, Karrer KM, Sun L, Manning G, Elde NC, Turkewitz AP, Asai DJ, Wilkes DE, Wang Y, Cai H, Collins K, Stewart BA, Lee SR, Wilamowska K, Weinberg Z, Ruzzo WL, Wloga D, Gaertig J, Frankel J, Tsao CC, Gorovsky MA, Keeling PJ, Waller RF, Patron NJ, Cherry JM, Stover NA, Krieger CJ, del Toro C, Ryder HF, Williamson SC, Barbeau RA, Hamilton EP, Orias E: Macronuclear genome sequence of the ciliate Tetrahymena thermophila, a model eukaryote. PLoS Biol 2006, 4:e286.

8. Salim HMW, Ring KL, Cavalcanti ARO: Patterns of codon usage in two ciliates that reassign the genetic code: Tetrahymena thermophila and Paramecium tetraurelia. Protist 2008, 159:283-298.

9. Tsukii Y: Evolution of mitochondrial DNA in Paramecium caudatum. Jpn J Genet 1994, 69:307-319.

10. Strüder-Kypke CM, Wright GA, Fokin SI, Lynn DH: Phylogenetic relationships of the subclass Peniculia (Oligohymenophorea, Ciliphora) inferred from small subunit rRNA gene sequences. J Eukaryot Microbiol 2000, 47:419-429.

11. Fokin SI, Przybos E, Chivilev SM, Beier CL, Horn M, Skotarczak B, Wodecka B, Fujishima M: Morphological and molecular investigations of Paramecium schewiakoffi sp. nov. (Ciliophora, Oligohymenophorea) and current status of distribution and taxonomy of Paramecium spp. Eur J Protistol 2004, 40:225-243.

12. Min XJ, Hickey DA: DNA barcodes provide a quick preview of mitochondrial genome composition. PLOS ONE 2007, 3:e325.

13. Clare EL, Kerr KCR, von Königslöw TE, Wilson JJ, Hebert PDN: Diagnosing mitochondrial DNA diversity: applications of a sentinel gene approach. J Mol Evol 2008, 66:362-367.

14. Barth D, Przybos E, Fokin SI, Schlegel M, Berendonk TU: Cytochrome $b$ data suggest rapid speciation within the Paramecium aurelia species complex. Mol Phylogenet Evol 2008, 49:669-673.

15. Catania F, Wurmser F, Potekhin AA, Przybos E, Lynch M: Genetic diversity in the Paramecium aurelia species complex. Mol Biol Evol 2009, 26:421-431.

16. Popescu CE, Lee RW: Mitochondrial genome sequence evolution in Chlamydomonas. Genetics 2007, 175:819-826.

17. Bernardi G: The human genome: organization and evolutionary history. Annu Rev Genet 1995, 29:445-476.

18. Galtier N, Lobry JR: Relationships between genomic G+C content, RNA secondary structures, and optimal group temperature in prokaryotes. J Mol Evol 1997, 44:632-636.

19. Przybos E, Fokin SI: Data on the occurrence of species of the Paramecium aurelia complex world-wide. Protistology 2000, 1:179-184.

20. Przybos E, Barth D, Berendonk TU: The Paramecium aurelia species complex, frequency and co-occurrence across Europe. Folia Biol Krakow 2008, 56:77-81.

21. Przybos E, Rautian M, Potekhin A: Species of the Paramecium aurelia complex in Russia, Lower Wolga Basin. Protistology 2005, 4:129-134.

22. Marais G: Biased gene conversion: implications for genome and sex evolution. Trends Ecol Evol 2003, 19:330-338

23. Tatarenkov A, Avise JC: Rapid concerted evolution in animal mitochondrial DNA. Proc R SOC B 2007, 274:822-826 
24. Smith DR, Lee RW: Mitochondrial genome of the colorless green alga Polytomella capuana: a linear molecule with an unprecedented GC content. Mol Biol Evol 2007, 25:487-496.

25. Jiang H, Guan W, Pinney D, Wang W, Gu Z: Relaxation of yeast mitochondrial functions after whole-genome duplication. Genome Res 2008, 18:1466-1471.

26. Gray MW, Lang BF, Cedergren R, Golding GB, Lemieux C, Sankoff D, Turmel M, Brossard N, Delage E, Littlejohn TG, Plante I, Rioux P, SaintLouis D, Zhu Y, Burger G: Genome structure and gene content in protist mitochondrial DNAs. Nucleic Acids Res 1998, 26:865-878.

27. Yang Z, Bielawski JP: Statistical methods for detecting molecular adaptation. Trends Ecol Evol 2000, 15:496-503.

28. Zhang Z, Li J, Zhao X-Q, Wang J, Wong G K-S, Yu J: KaKs_Calculator: Calculating $\mathrm{Ka}$ and $\mathrm{Ks}$ though model selection and model averaging. Geno Prot Bioinf 2006, 4:259-263.

29. Lunt DH, Whipple LE, Hyman BC: Mitochondrial DNA variable number tandem repeats (VNTRs): utility and problems in molecular ecology. Mol Ecol 1998, 7:1441-1455.

30. Martin FN, Bensasson D, Tyler BM, Boore JL: Mitochondrial genome sequences and comparative genomics of Phytophthora ramorum and $P$. sojae. Curr Genet 2007, 51:285-296.

31. Karlin S, Burge C: Trinucleotide repeats and long homopeptides in genes and proteins associated with nervous system disease and development. Proc Natl Acad Sci USA 1996, 93:1560-1565.

32. Jordan P, Snyder LAS, Saunders NJ: Diversity in coding tandem repeats in related Neisseria spp. BMC Microbiol 2003, 3:23

33. Verstrepen KJ, Jansen A, Lewitter F, Fink GR: Intragenic tandem repeats generate functional variability. Nat Genet 2005, 37:986-990.

34. Bois $\mathrm{P}$, Jeffreys AJ: Minisatellite instability and germline mutation. Cell Mol Life Sci 1999, 55:1636-1648.

35. Barth D, Krenek S, Fokin SI, Berendonk TU: Intraspecific genetic variation in Paramecium, revealed by mitochondrial cytochrome c oxidase I sequences. J Eukaryot Microbiol 2006, 53:20-25

36. Chantangsi C, Lynn DH, Brandl MT, Cole JC, Hetrick N, Ikonomi P: Barcoding ciliates: a comprehensive study of 75 isolates of the genus Tetrahymena. Int J Syst Evol Microbiol 2007, 57:2412-2425.

37. Wesley UV, Wesley CS: Rapid directional walk within DNA clones by stepout PCR. Methods Mol Biol 1997, 67:279-285.

38. Antal Z, Rascle C, Fevre M, Bruel C: Single oligonucleotide nested PCR: a rapid method for the isolation of genes and their flanking regions from expressed sequence tags. Curr Genet 2004, 46:240-246.

39. Chen $X$, Wu R: Direct amplification of unknown genes and fragments by Uneven polymerase chain reaction. Gene 1997, 185:195-199.

40. Hall TA: BioEdit: a user-friendly biological sequence alignment editor and analysis program for Windows 95/98/NT. Nucleic Acids Res Symp Ser 1999, 41:95-98.

41. Berriman $M$, Rutherford $K$ : Viewing and annotating sequence data with Artemis. Brief Bioinformatics 2003, 4:124-132.

42. Altschul SF, Madden TL, Schäffer AA, Zhang J, Zhang Z, Miller W, Lipman DJ: Gapped BLAST and PSI-BLAST: a new generation of protein database search programs. Nucleic Acids Res 1997, 25:3389-3402.

43. Lowe TM, Eddy SR: tRNAscan SE: a program for improved detection of transfer RNA genes in genomic sequence. Nucleic Acids Res 1997, 25:955-964.

44. Tamura K, Dudley J, Nei M, Kumar S: MEGA4: Molecular evolutionary genetics analysis (MEGA) software version 4.0. Mol Biol Evol 2007 24:1596-1599.

45. Stothard P: The Sequence Manipulation Suite: JavaScript programs for analyzing and formatting protein and DNA sequences. BioTechniques 2000, 28:1102-1104.

46. Wright F: The 'effective number of codons' used in a gene. Gene 1990 87:23-29.

47. Goldman N, Yang Z: A codon-based model of nucleotide substitution for protein-coding DNA sequences. Mol Biol Evol 1994, 11:725-736.

doi:10.1186/1471-2164-12-272

Cite this article as: Barth and Berendonk: The mitochondrial genome sequence of the ciliate Paramecium caudatum reveals a shift in nucleotide composition and codon usage within the genus Paramecium. BMC Genomics 2011 12:272

\section{Submit your next manuscript to BioMed Central and take full advantage of:}

- Convenient online submission

- Thorough peer review

- No space constraints or color figure charges

- Immediate publication on acceptance

- Inclusion in PubMed, CAS, Scopus and Google Scholar

- Research which is freely available for redistribution 